\title{
Neural mechanisms of mindfulness meditation: bridging clinical and neuroscience investigations
}

\author{
Anne Maj van der Velden and Andreas Roepstorff
}

Tang, Hölzel and Posner have written an excellent Review of the literature on the neural correlates and mechanisms of mindfulness meditation (The neuroscience of mindfulness meditation. Nat. Rev. Neurosci. 16, 213-225 (2015) $)^{1}$, and they provide much needed methodological recommendations for future research. However, on the basis of the overview presented, it has become apparent that there is a gap between the clinical and neuroscientific branches of research on mindfulness meditation.

Although most clinical evidence has been gathered from two interventions that incorporate mindfulness meditation (mindfulness-based stress reduction (MBSR) and mindfulness-based cognitive therapy $\left.(\mathrm{MBCT})^{2}\right)$, research on the neural correlates of MBSR and MBCT and the prediction of clinical outcomes is still extremely limited. Take, for instance, MBCT, which was developed as a preventive treatment for recurrent depression. Evidence for the efficacy of MBCT has increased rapidly over the past decade, and MBCT has been subject to rigorous clinical investigation in large randomized controlled trials $s^{3-5}$. As a result, MBCT is now recommended in several national clinical guidelines, such as those of the UK National Institute for Health and Clinical Excellence ${ }^{6}$, as a preventive treatment for recurrent depression. However, how MBCT treatment leads to reduced risk of depressive relapse or recurrence is still yet to be fully elucidated, and the underlying neural mechanisms are particularly poorly understood. In fact, in the rapidly growing literature on the neural mechanisms of mindfulness meditation, only two studies have specifically looked at the neural mechanisms of MBCT in the treatment of recurrent major depressive disorder ${ }^{7}$.

We find it remarkable that few neuroscientific investigations of mindfulness meditation have focused on the interventions that have shown the most clinical promise. By contrast, many of the mindfulness interventions cited in the Review by Tang, Hölzel and Posner ${ }^{1}$ have been subject to only limited research on clinical and well-being outcomes, and few have been investigated in large rigorous clinical trials. As previously called for, tighter integration and collaboration between the clinical and neuroscientific branches of research are needed ${ }^{8}$. Clinicians and neuroscientists need to work closer together to understand and improve the clinical potential of mindfulness-based treatments.

Anne Maj van der Velden and Andreas Roepstorff are at the Interacting Minds Centre, Aarhus University, 8000 Aarhus, Denmark.

Anne Maj van der Velden is also at the Danish Center for Mindfulness, Aarhus University Hospital, 8000 Aarhus, Denmark.

Correspondence to A.M.V.D.V. e-mail:wrp704@alumni.ku.dk doi: 10.1038/nrn3916-c1 Published online 17 June 2015

1. Tang, Y.-Y., Hölzel, B. K. \& Posner, M. I. The neuroscience of mindfulness meditation. Nat. Rev. Neurosci. 16, 213-225 (2015).

2. Khoury, B. et al. Mindfulness-based therapy: a comprehensive meta-analysis. Clin. Psychol. Rev. 33, 763-771 (2013)

3. Kuyken, W. et al. Effectiveness and cost-effectiveness of mindfulness-based cognitive therapy compared with maintenance anti-depressant treatment in the prevention of depressive relapse/recurrence: a randomised controlled trial. Lancet http://dx.doi org/10.1016/S0140-6736(14)62222-4 (2015).

4. Piet, J. \& Hougaard, E. The effect of mindfulnessbased cognitive therapy for prevention of relapse in recurrent major depressive disorder: a systematic review and meta-analysis. Clin. Psychol. Rev. 31, 1032-1040 (2011)

5. Williams, J. M. et al. Mindfulness-based cognitive therapy for preventing relapse in recurrent depression a randomized dismantling trial. J. Consult. Clin. Psychol. 82, 275-286 (2014).

6. National Collaborating Centre for Mental Health. Depression in adults: the treatment and management of depression in adults. Ch. 1.9. NICE [online], http:// www.nice.org.uk/guidance/cg90/chapter/1recommendations\#continuation-and-relapse prevention (2009).

7. van der Velden, A. M. et al. A systematic review of mechanisms in mindfulness-based cognitive therapy in the treatment of recurrent depressive disorder. Clin. Psychol. Rev. 37, 26-39 (2015).

8. Holmes, E. M., Craske, M. G. \& Graybiel, A. M. A call for mental-health science. Nature 511, 287-289 (2014).

Competing interests statement The authors declare no competing interests. 